\title{
Du pénétromètre dynamique au battage des pieux
}

\section{H. GONIN}

SIMECSOL

8, avenue Newton, 92350 Le Plessis-Robinson

L'auteur poursuit dans cet article une analyse dont il avait rendu compte dans le $n^{2} 9$ de la $R F G$ en 1979 concernant le battage des pieux.

Il s'attache, après avoir décrit de quelle manière se transmet et s'amortit dans le sol l'énergie d'un choc d'une masse cylindrique sur une tige, à définir un modèle simple adapté aux pénétromètres dynamiques et ne faisant intervenir que deux paramètres pour définir le sol.

La prise en compte d'une succession de couches de sol peut se faire à l'aide d'un tableur et est donc à la portée de tout technicien sans nécessité de faire appel à un logiciel de prévision de battage. Un exemple schématique est donné.

Enfin, à la lumiẻre de cette analyse, une critique constructive est faite des formules de battage des pieux et une amorce de méthodologie est donnée pour des observations sur chantier permettant d'apprécier la résistance dynamique au battage si l'on connaît le rendement du mouton et quand on ne dispose pas de mesures directes de l'onde de choc dans le pieu.

\section{Dynamic penetration test and pile driving analysis}

In this paper, the author follows up an analysis made in an earlier paper on pile driving by the use of a hammer (RFG $n^{2}$ 9, 1979).

After discussing the transmission and damping of the stresswave induced by the impact, a simple model is proposed with only two parameters for describing the soil. This model is adapted to dynamic penetration.

Successive layers of soil are modelled using a spreadsheet. enabling technicians to work without using a driving program. A schematic example is given. At last, in the light of the analysis of dynamic penetration, driving formulae are discussed. Further, a methodology is suggested for the analysis of the dynamic resistance on site when knowing the hammer output and under the condition that the stress wave is not available by direct measurements. 
Dans le domaine de la géotechnique, comme dans d'autres, le conformisme, le poids des habitudes, ou, au contraire, le modernisme érigé en système de pensée, sont à l'origine de querelles d'écoles dans lesquelles les idées toutes faites prévalent trop souvent sur l'observation et la logique cartésienne. Or l'ingénieur se heurte aux réalités concrètes, et il a le plus grand besoin de concepts clairs et d'idées justes sur lesquels baser un raisonnement pour vérifier la validité d'une démarche ou comprendre le fondement des disparités entre prévisions et observations. Il lui faut aussi disposer, pour son analyse, de modèles simples, débarrassés des paramètres inutiles et représentant correctement les phénomènes essentiels. C'est lorsqu'il possède de tels outils qu'il peut, l'expérience aidant, acquérir un précieux savoir-faire, qui s'appuie sur des études paramétriques bien choisies.

On propose, dans ce qui suit, un modèle élémentaire pour les pénétromètres dynamiques, appareils improprement décriés lorsque l'on confond l'outil et la mauvaise utilisation qui peut en être faite. Le modèle vaut pour le battage des pieux. Cette étude prolonge des travaux antérieurs publiés dans les Annales de I'TTBTP (Gonin, 1978) et dans la Revue Française de Géotechnique (Gonin, 1979).

\section{1}

\section{Trois questions simples}

Pour le pénétromètre statique, le fonçage vertical d'une pointe à vitesse constante engendre dans le sol une réaction complexe qui intéresse le comportement non linéaire du milieu, jusqu'à atteindre le domaine des écoulements plastiques. L'interprétation de cet essai est donc basée sur des observations directes et des corrélations, plus que sur des considérations théoriques, abordées récemment seulement par les recherches.

Pour le pénétromètre dynamique, il ne s'agit plus d'un effort de fonçage appliqué à vitesse contrôlée, mais d'une énergie connue (choc d'une masse frappante) communiquée à une tige, dont une part est dissipée dans le sol et une autre utilisée pour l'enfoncement. Bien que la sollicitation soit tout aussi complexe que pour le fonçage quasi statique, trois questions simples viennent à l'esprit:

- comment se transmet l'énergie de la masse frappante à la tige?

- comment cette énergie se dissipe-t-elle dans le sol? - comment est-elle utilisée pour l'enfoncement?

\section{1}

\section{Transfert de l'énergie}

Prenons le cas le plus simple: la masse frappante et la tige sont des cylindres élastiques de même section; on ne quitte pas le domaine élastique et les ondes engendrées par le choc sont des ondes de compression planes. Les notations sont explicitées sur la figure 1; indiquons seulement que la célérité des ondes est notée $\mathrm{c}$, alors que les vitesses particulaires de la matière sont indiquées $\mathrm{V}$ ou $\mathrm{V}_{\mathrm{p}}$.

La figure 1 illustre la situation:

1. avant le choc, masse frappante immobile;
2. à l'instant origine, lorsque la masse frappante arrive au contact de la tige avec une vitesse $\mathrm{V}_{\mathrm{M}}=(2 \mathrm{gH})^{1 / 2}$;

3. au temps $t=d t$ après le premier contact $(\mathrm{dt}<\mathrm{h} / \mathrm{c})$;

4. au temps $t=t(t>2 h / c)$.

Au temps $t=d t$, le déplacement au sommet de la masse frappante depuis l'origine est $\Delta \mathrm{l}=\mathrm{V}_{\mathrm{M}} \mathrm{dt}$, puisque le front de l'onde ne l'a pas atteint; les portions de tige et de masse frappante parcourues par l'onde de compression ont la même longueur cdt, soit une partie comprimée de longueur 2 cdit; donc:

- le déplacement du sommet de la tige, seule section mise en mouvement depuis l'origine du choc, est égal à $\left(V_{M} / 2\right) d t$, puisque c'est le milieu de la partie comprimée élastiquement dont l'extrémité basse a eu un déplacement nul et l'extrémitẻ haute un déplacement égal à $\mathrm{V}_{\mathrm{M}} \mathrm{dt}$ :

- les fronts d'onde se déplaçant à vitesse constante $\left[\mathrm{c}=(\mathrm{E} / \mathrm{\rho})^{1 / 2}\right]$, la vitesse particulaire dans toute la partie comprimée est égale à $V_{M} / 2$; la contrainte (intensité de londe) est telle que:

soit:

$$
2 \operatorname{cdt}(\sigma / E)=V_{M} d t=2 V_{p} d t
$$

ou encore: $\sigma=V_{p} \frac{E}{c}=\rho c V_{p}$ formule générale de l'équilibre local.

L'énergie de l'onde est la somme de l'énergie de compression et de l'énergie cinétique, qui sont égales: $\xi=1 / 2\left(2\right.$ a cdt $\left.\sigma^{2} / E\right)+1 / 2\left(2\right.$ pacdt $\left.V_{p}{ }^{2}\right)=1 / 2$ pacdt $V_{M}{ }^{2}$

Cette énergie devient égale à celle de la masse frappante à l'instant origine lorsque $\mathrm{dt}=\mathrm{h} / \mathrm{c}$; la longueur de l'onde est alors $2 \mathrm{~h}=2 \mathrm{~L}$ ( $\mathrm{L}$ correspond à la longueur de tige de même masse que la masse frappante, paramètre qui nous sera utile par la suite). Le temps du transfert d'énergie dans la tige dure donc $2 \mathrm{~h} / \mathrm{c}=2 \mathrm{~L} / \mathrm{c}$. Après ce temps, l'énergie est véhiculée dans la tige sous forme d'une onde "créneau» de compression de longueur $2 \mathrm{~L}=2 \mathrm{~h}$, d'intensité $\sigma=(\mathrm{E} / \mathrm{c})(\mathrm{V} / \mathrm{L})$, se déplaçant vers le bas avec une célérité $c=(E / p)^{1 / 2}$. Le raccourcissement $\Delta \mathrm{L}$ dû̀ à cette onde est égal à $\mathrm{V}_{\mathrm{M}}(\mathrm{L} / \mathrm{c})$; la vitesse particulaire de la tige au passage de l'onde est $V_{M} / 2$. Si l'on fait intervenir l'impédance de la tige $\mathrm{Z}=$ apc prise comme unité, le raccourcissement de l'onde $\Delta \mathrm{L}$ mesure la quantité de mouvement:

$$
\Delta \mathrm{L}=(\mathrm{L} / \mathrm{c}) \mathrm{V}_{\mathrm{M}}=\mathrm{Lap} \mathrm{V}_{\mathrm{M}} / \mathrm{apc}=\mathrm{MV}_{\mathrm{M}} / \mathrm{Z}
$$

Somme toute, la réponse à la première question pourrait être:

Le transfert d'énergie se fait sous forme d'une onde de compression de célérité $c=(E / \rho)^{1 / 2}$ dont l'énergie de compression et l'énergie cinétique sont égales. Le raccourcissement au passage de l'onde mesure la quantité de mouvement avec comme unité limpédance de la tige.

\section{2}

\section{Dissipation de l'énergie dans le sol: amortissement}

Que devient cette onde lorsque la tige est au contact du sol? Elle perd une part de son énergie (amortissement). Elle perd de l'intensité et donc de la vitesse particulaire, mais sans modification de la célérité, qui ne dépend que de la matière de la tige. C'est la mobilisation de la résistance au cisaillement entre le sol et la tige 


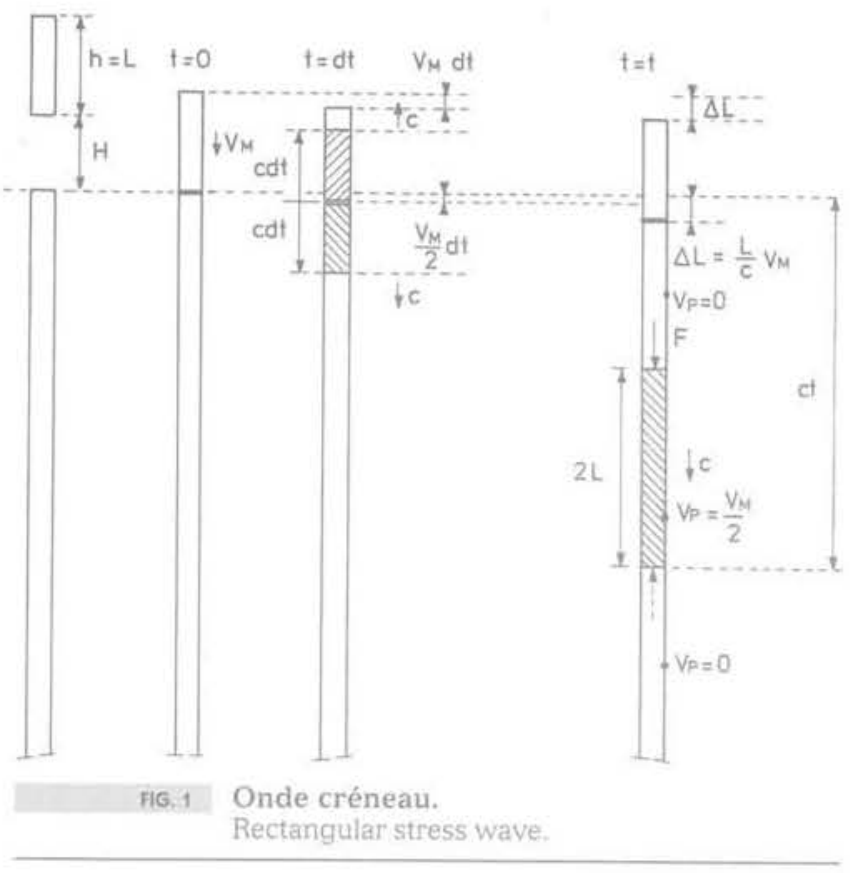

qui est responsable de cette perte. Mais on est loin d'une vitesse de cisaillement de $2 \mathrm{~cm} / \mathrm{s}$ comme dans le pénétromètre statique; l'ordre de grandeur des vitesses particulaires de la tige au passage de l'onde est plutôt de 1 à $6 \mathrm{~m} / \mathrm{s}$, c'est-à-dire une à plusieurs centaines de fois plus élevée.

Une hypothèse simple est que la contrainte de cisaillement mobilisée est proportionnelle à la vitesse de déplacement de la tige par rapport au sol (vitesse de cisaillement); on a alors, pour une longueur $\Delta z$ de tige, en écrivant que les forces résistantes sont proportionnelles à la surface en contact avec le sol et à la vitesse particulaire de la tige :

$\mathrm{p}=$ périmètre frottant:

$$
-\mathrm{K} . \mathrm{p} \cdot \Delta z(\mathrm{c} / \mathrm{E}) \sigma=\Delta \sigma \cdot \mathrm{a}
$$

$\mathrm{K}=$ facteur constant, ayant les dimensions d'une contrainte par unité de vitesse.

La diminution de la contrainte (intensité) de l'onde en fonction de la fiche dans un sol homogène est alors exponentielle:

$$
\sigma(\mathrm{z})=\sigma_{0} \exp [-\mathrm{K}(\mathrm{p} / \mathrm{a})(\mathrm{c} / \mathrm{E}) \mathrm{z}]=\sigma_{0} \exp [-\mathrm{Kpz} / \mathrm{Z}]
$$

$\mathrm{Z}=$ impédance de la tige $=\mathrm{E} a / \mathrm{c}=$ apc.

L'hypothèse de base diffère de celle du frottement de Coulomb; c'est pourquoi on peut en discuter le bien-fondé dans certains cas; il importe donc de bien comprendre quelle en est la signification physique.

Physiquement, cela revient à supposer que dans le domaine des vitesses de cisaillement concerné, une fois rompue les liaisons initiales du contact tige-sol, la résistance au cisaillement mobilisée au contact tige-sol est proportionnelle à cette vitesse; sur le diagramme de la figure 2, supposé se rapporter à une argile saturée, on aurait par exemple:

- pour une vitesse nulle: $\tau_{1}=\mathrm{C}_{\mathrm{u}}$;

- pour une vitesse de $2 \mathrm{~cm} / \mathrm{s}$ (pénétromètre statique) : $\tau=\mathrm{C}$ résiduel ;

- pour une vitesse plus importante, non précisée: $\tau_{1}=\mathrm{C}$ remanié:

- pour notre plage de vitesse (1 à $6 \mathrm{~m} / \mathrm{s}$ ) : $\tau_{1}$ proportionnel à $\mathrm{V}$.

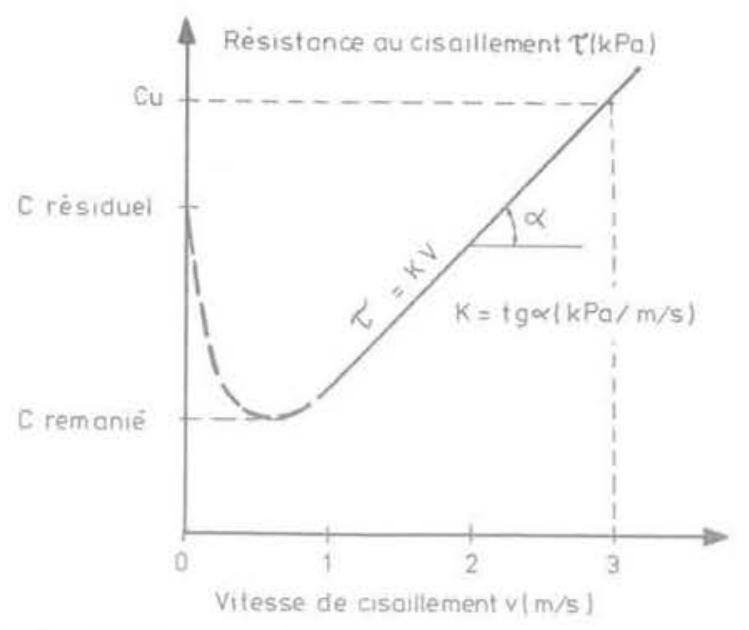

FiG. 2 Facteur d'amortissement $\mathrm{K}$. Dynamic factor $\mathrm{K}$.

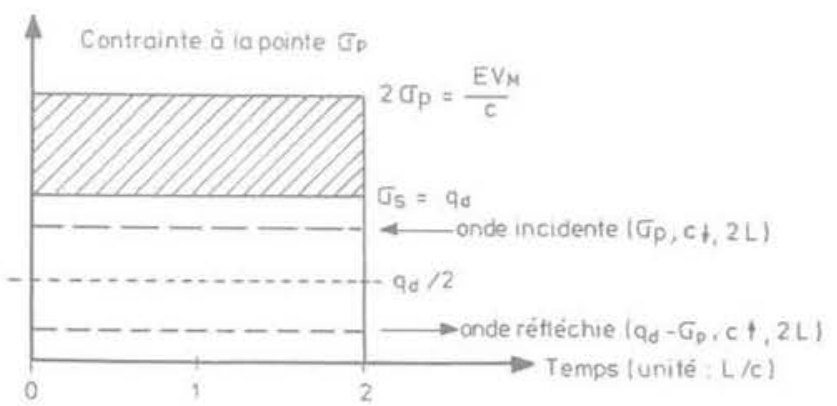

FG.3 Enfoncement à la pointe. Tip penetration.

Si notre hypothèse représente valablement le phénomène prépondérant, nous pourrons caractériser le sol par un seul paramètre, K. Notre deuxième question trouve là une réponse sous condition de vérification: L'amortissement de l'onde dans le sol est proportionnel à la vitesse particulaire (ou à l'intensité = contrainte de compression de l'onde).

\section{3}

\section{Enfoncement dans le sol}

Abordons la troisième question. Reprenons notre onde créneau; elle arrive à l'extrémité basse de la tige. Si cette tige est dans le vide, l'onde se réfléchit en onde d'intensité et de célérité opposées à celles de l'onde incidente: à l'extrémité, la contrainte est nulle, et la vitesse particulaire est donc doublée. Cela dure le temps nécessaire à l'onde incidente pour parcourir cette extrémité, soit $2 \mathrm{~L} / \mathrm{c}$.

La vitesse particulaire étant $\left(V_{M} / 2\right) .2=V_{M}$, le déplacement sera $2 \mathrm{~L} .\left(\mathrm{V}_{\mathrm{M}} / \mathrm{c}\right)$, le double de la compression $\Delta \mathrm{L}$. de la tige au passage de l'onde. Il y a détente du ressort comprimé, qui se met en traction.

Si la tige repose sur un milieu totalement incompressible, la vitesse particulaire doit être nulle à l'extrémité. C'est donc la contrainte de compression qui est 
doublée: $\sigma_{p}=(\mathrm{E} / \mathrm{c}) \mathrm{V}_{\mathrm{M}}$. L'onde est réfléchie en onde de compression égale à londe incidente et de sens inverse.

Dans ces deux cas, l'énergie de l'onde est totalement réfléchie, ce qui ne doit pas nous étonner, l'énergie de l'onde ne dépendant pas du signe de son intensité, et aucune énergie n'étant perdue en pointe (dans un cas, force nulle; dans l'autre, déplacement nul).

Si la tige s'enfonce à son extrémité basse, une hypothèse simple est que la réaction du sol est rigide plastique: l'onde est réfléchie en onde de compression jusqu'à un certain seuil, en onde de décompression audelà. Si ce seuil correspond à une contrainte $\sigma_{c}=q_{0}$, en supposant que $\mathrm{q}_{\mathrm{d}}$ est inférieur à $2 \sigma_{\mathrm{g}}=(\mathrm{E} / \mathrm{c}) \mathrm{V}_{\mathrm{M}}$, la vitesse particulaire à l'extrémité sera proportionnelle à $2 \sigma_{p}-q_{d a}$ :

$$
V_{p}=\frac{C}{E}\left(2 \sigma_{p}-q_{e}\right)=\frac{C}{E}\left(\frac{E V_{M}}{C}-q_{d}\right)
$$

L'enfoncement ayant une durée égale à $2 \mathrm{~L} / \mathrm{c}$, il sera égal à : $s=\frac{2 L}{E}\left(\frac{E V_{M}}{c}-q_{d}\right)$

Autrement dit, sur la figure 3 , ce sera au facteur c/E près, l'aire hachurée correspondant à un "écrêtement du double de l'onde incidente.

Voilà une réponse à notre troisième question, réponse sous condition également.

La réaction du sol en pointe est rigide plastique. Un seul paramètre suffit à la définir, la résistance dynamique en pointe $q_{d}$. L'énergie utilisée pour la pénétration est égale à la différence entre l'énergie de l'onde incidente et celle de l'onde réfléchie:

$$
\mathrm{aq}_{\mathrm{d}} \mathrm{s}=\frac{2 \mathrm{aL} \mathrm{q}_{\mathrm{d}}}{\mathrm{E}}\left(2 \sigma_{p}-\mathrm{q}_{\mathrm{d}}\right)=\frac{2 \mathrm{aL} \sigma_{\mathrm{p}}^{2}}{\mathrm{E}}-\frac{2 \mathrm{aL}}{\mathrm{E}}\left(\mathrm{q}_{\mathrm{i}}-\sigma_{\mathrm{p}}\right)^{z}=\xi_{\mathrm{i}}-\xi_{\mathrm{p}} .
$$

\section{2}

\section{Trois questions de plus}

Tout ce que nous avons vu pour une onde créneau peut être généralisé pour une onde de forme quelconque, dont l'intensité n'est pas constante pendant son passage. Mais nous ne pourrons pas aller plus loin sans examiner trois nouveaux problèmes:

- que se passe-t-il si la masse frappante est un cylindre de section $\mathrm{A}$ différente de celle de la tige: $\mathrm{A}=$ ra? - que se passe-t-il lorsqu'une onde créneau parcourant une tige rencontre un changement de section?

- que se passe-t-il si on interpose entre la masse frappante et la tige une enclume (également cylindrique, et d'une autre section droite)?

En effet, si l'on revient au pénétromètre dynamique qui nous occupe, ces trois questions se posent (voir Fig. 4).

\section{1}

\section{Choc d'un mouton cylindrique de section différente de la tige}

La réponse à la première question a été donnée par Barre de Saint-Venant dans un mémoire célèbre (SaintVenant, 1867). Comment est-il possible que nous l'ayons oublié pendant des décennies, au point de privilégier un raisonnement (formule des Hollandais) qui n'a aucun fondement physique valable?
Si l'on admet que les ondes générées sont planes, ce qui est une approximation, le choc de la masse sur la tige induit dans celle-ci une succession d'ondes-créneaux ayant toutes une longueur égale à deux fois la hauteur de la masse frappante, dont les intensités forment une série géométrique décroissante de raison $(r-1) /(r+1)$, la valeur initiale étant $(E / c) V_{M} r /(1+r)$ (Fig. 5). On voit que pour $r=1, \sigma_{1}=(E / c)\left(V_{M} / 2\right)$ et toutes celles de rang supérieur à un sont nulles, la raison de la série étant nulle.

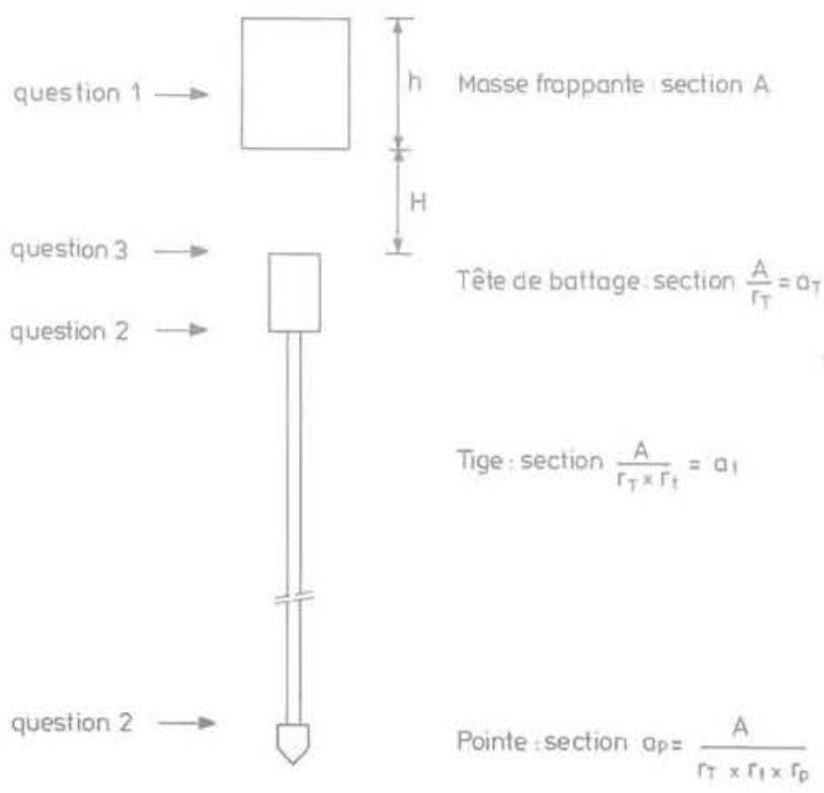

FIG.4 Pénétromètre dynamique. Dynamic penetrometer.

Lorsque r tend vers zéro, l'intensité de l'onde tend vers une fonction exponentielle décroissante du temps écoulé depuis l'origine du choc:

$$
\sigma_{0} \exp (-\mathrm{ct} / \mathrm{L})=(\mathrm{E} / \mathrm{c}) \mathrm{V}_{\mathrm{M}} \exp (-\mathrm{ct} / \mathrm{L}) \text {. }
$$

$\mathrm{L}$ étant la longueur de tige ayant la même masse que la masse frappante; rappelons que dans notre cas simple de la première partie, cette longueur est aussi celle de la masse frappante.

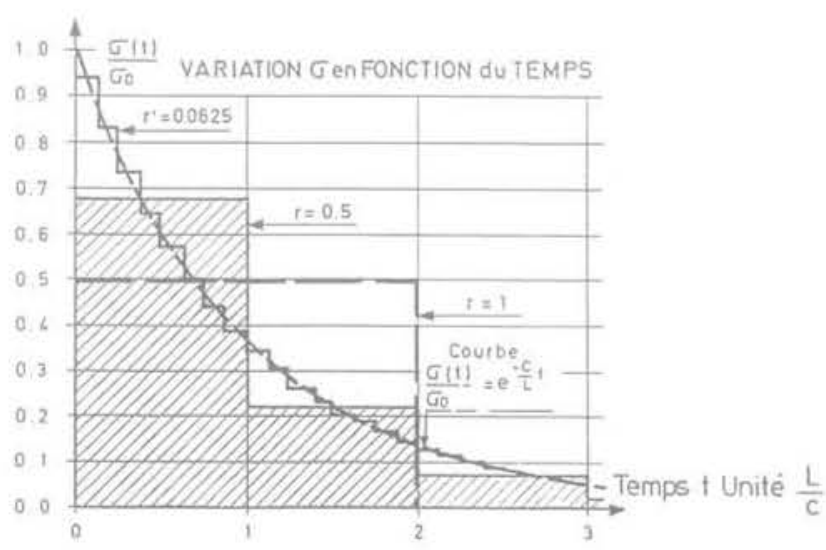

FIG. 5 Choc d'une masse sur une tige. Mass impact on a drill string. 
Dans tous les cas, le raccourcissement $\Delta \mathrm{L}$ dû à la compression de l'onde à la même expression: $\Delta \mathrm{L}=$ $\mathrm{L}\left(\mathrm{V}_{\mathrm{M}} / \mathrm{c}\right.$ ) (lorsque l'enfoncement devient nul, le $\alpha$ refus élastique » devient égal à $2 \Delta \mathrm{L}$ ).

Dans le cas où r est petit, le pourcentage d'énergie transmise au temps $\mathrm{t}=\mathrm{L} / \mathrm{c}$, lorsque l'onde a parcouru la longueur L, est donné par: $1-\left(\sigma_{1} / \sigma_{0}\right)^{2}=1-\exp (-2 \mathrm{ct} / \mathrm{L})$. soit $86 \%$. Au temps $t=\mathrm{L} / 2 \mathrm{c}$, soit lorsque l'onde a parcouru la distance $L / 2$, ce pourcentage est $63 \%$. En terme de transmission d'énergie, l'essentiel est déjà joué !

Compte tenu des observations faites en première partie, il est facile maintenant de résoudre complètement le problème de l'enfoncement dans le sol dú à la première onde incidente lorsque r est petit. En effet, le même raisonnement (voir Fig. 6) conduit à l'expression suivante pour la pénétration s due à une onde dont la contrainte de pic (initiale) est $\sigma_{\mathrm{a}}$ :

$$
\mathrm{s}=2 \frac{\sigma_{\mathrm{a}} \mathrm{L}}{\mathrm{E}}\left[1+\frac{\mathrm{q}_{\mathrm{d}}}{2 \sigma_{\mathrm{a}}}\left(\ln \frac{\mathrm{q}_{\mathrm{d}}}{2 \sigma_{\mathrm{a}}}-1\right)\right]
$$

Par ailleurs, la contrainte de pic amortie $\sigma_{\mathrm{a}}$ se déduit de la contrainte de pic initiale $\sigma_{\sigma}$, si le sol est homogène et a un facteur d'amortissement $\mathrm{K}$, à l'aide de la relation: $\sigma_{\mathrm{a}}=\sigma_{0} \exp \left[-\mathrm{K} \frac{\mathrm{p}}{\mathrm{Z}} \mathrm{z}\right]$, si r est très petit: $\sigma_{0}=\frac{E}{c} V_{M}$.

$\mathrm{Z}=$ impédance de la tige $: \mathrm{Ea} / \mathrm{c}=\mathrm{apc}$.

Comme nous l'avons déjà remarqué, l'enfoncement s est, à un facteur c/E près, égal à l'aire hachurée sur la figure 6: c'est en effet le produit de la vitesse particulaire en pointe par le temps écoulé pendant l'enfoncement, et la vitesse particulaire est proportionnelle à la contrainte $\sigma$.

Remarquons que la compression due à l'onde réfléchie, ou refus élastique mesuré au sommet de la tige, s'exprime facilement:

$$
\mathrm{s}_{e}=\frac{q_{d}}{E}\left(-\ln \frac{q_{d}}{2 \sigma_{a}}+1\right)
$$

Si l'on connait les paramètres de l'onde $\left(\sigma_{\sigma} L\right)$ et les valeurs de $s$ et $s_{\mathrm{e}}$ (facilement mesurables), on peut en déduire l'amortissement de l'onde et la résistance dynamique de pointe. Nous reviendrons sur ce point plus avant.

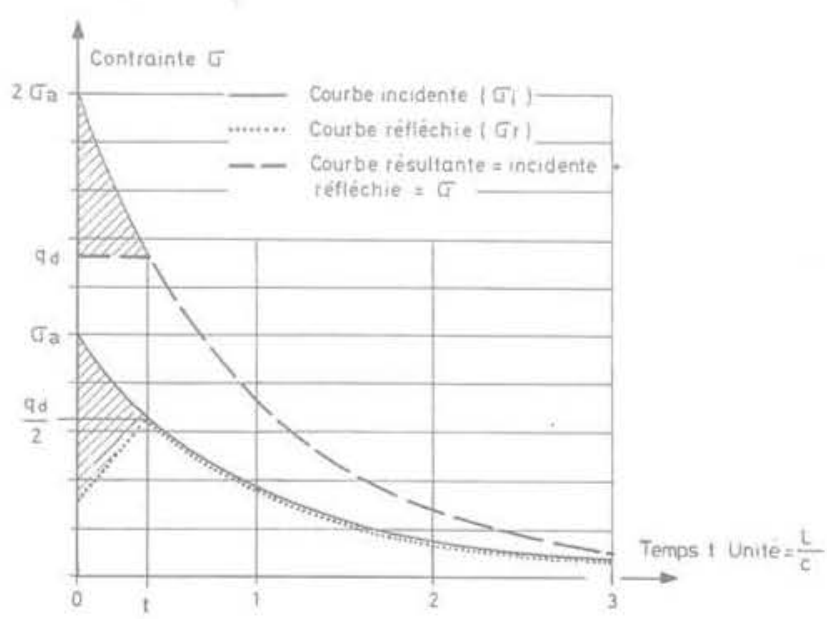

FG.6 Enfoncement en pied. Toe penetration.
On voit bien que l'onde est caractérisée par son intensité (contrainte de pic) et son énergie (longueur); deux paramètres suffisent à la définir, de même que pour la masse frappante, il suffit de connaitre sa masse et sa vitesse à l'impact. La réponse à la première question est donc: Le transfert se fait par un empilement continu d'ondes créneaux dont les intensités suivent une série géométrique décroissante. Les principes énoncés précédemment sont conservés.

\section{2}

\section{Changement de section: transmission et réflexion}

Abordons maintenant la deuxième question, qui porte sur l'incidence d'un changement de section sur une onde incidente. Elle est simple à résoudre si l'on a bien assimilé ce qu'est une onde. Au changement de section, il y a discontinuité des vitesses particulaires et des contraintes associées, avec transmission et réflexion; moyennant des hypothèses précises sur les signes, rappelées dans la figure 7 , on a les formules suivantes, valables dans tous les cas:

- pour les vitesses particulaires:

$$
V_{t}=\frac{2 r}{1+r} V_{i} \quad V_{r}=\frac{r-1}{1+r} V_{i}
$$

- pour les contraintes associées:

$$
\sigma_{t}=\frac{2 r}{1+r} \sigma_{i} \quad \sigma_{r}=\frac{1-r}{1+r} \sigma_{i}
$$

$$
\begin{aligned}
& \text { Onde de compression vers le bas } \\
& v p=v \text {. } \\
& x, v(f+c) \quad v>0 \quad G>0 \quad c=|c| \\
& v p=0 \\
& \text { Onde de compression vers le haut } \\
& v p=0 \\
& -I, v i+c|\quad v<0 \quad \sigma>0 \quad c=-| c \mid \\
& v p=v i \\
& \text { Onde de reláchement vers le bas } \\
& 4 p=v+ \\
& \stackrel{+}{*}, v(i c) \quad v<0 \quad G<0 \quad c=|c| \\
& v p=0
\end{aligned}
$$

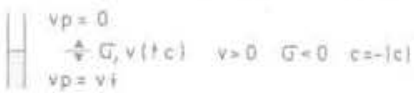

FIG. 7 Conventions de signes. Sign conventions.

On remarquera qu'il y a un changement de signe entre les vitesses particulaires et les contraintes pour les ondes réfléchies.

\section{3}

\section{Incidence d'une enclume (tête de battage)}

La troisième question pose un problème facile dans son principe mais fastidieux à résoudre. Nous l'avons fait dans un cas particulier avec un élève de l'École Centrale, Pascal Bonfort, en 1978. Le résultat est assez surprenant, car le contact entre la masse frappante et la tête de battage ne dure qu'un dix-millième de seconde 
tous les millièmes de seconde ! On peut voir que dans le cas étudié, la contrainte de pic initiale dans la tige est multipliée par 1,5 par la tête de battage (voir Fig. 8 et 9).

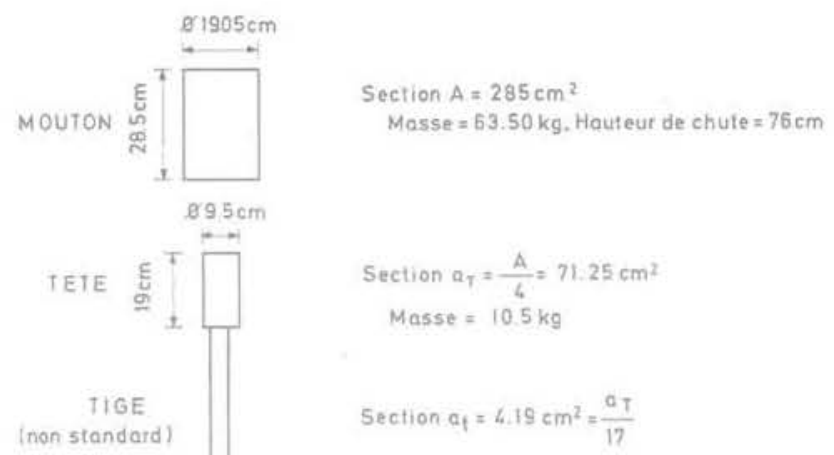

FIG. Données prises en compte pour l'étude de l'influence d'une tête.

Data taken into account for the influence of the cap.

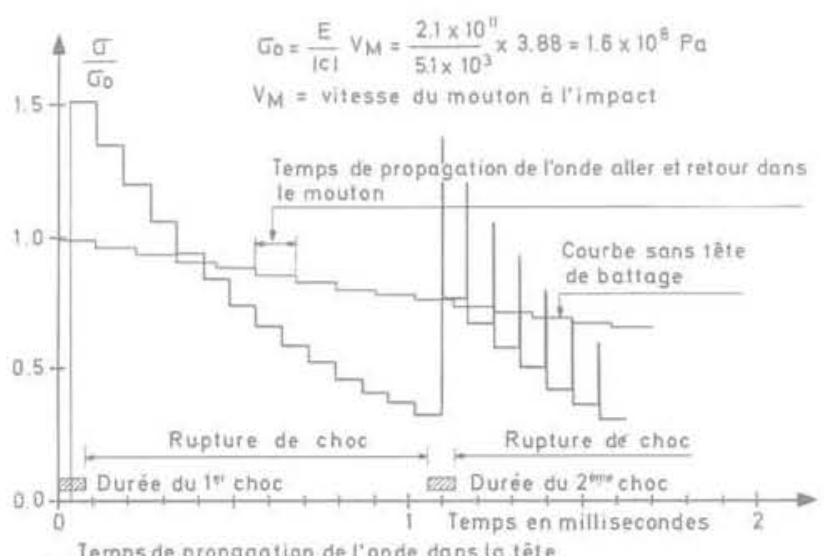

Temps de propagation de l'onde dans ta tête

FIG.9 Onde de contrainte au sommet de la tige munie d'une tête.

Stress wave at the top of the drill string.

De ce fait, le transfert d'énergie est plus intense au début, pour se trouver au contraire retardé après un millième de seconde (ce qui correspond, dans ce cas, à L/3) (voir Fig, 10). Il est indiscutable que la présence de la tête, en allongeant la «queue » de l'onde, augmente un peu la part qui ne sera plus utilisable pour l'enfoncement, parce que trop peu intense et amortie par les multiples réflexions qu'elle aura subie dans son trajet. Par contre, l'intensité initiale est augmentée.

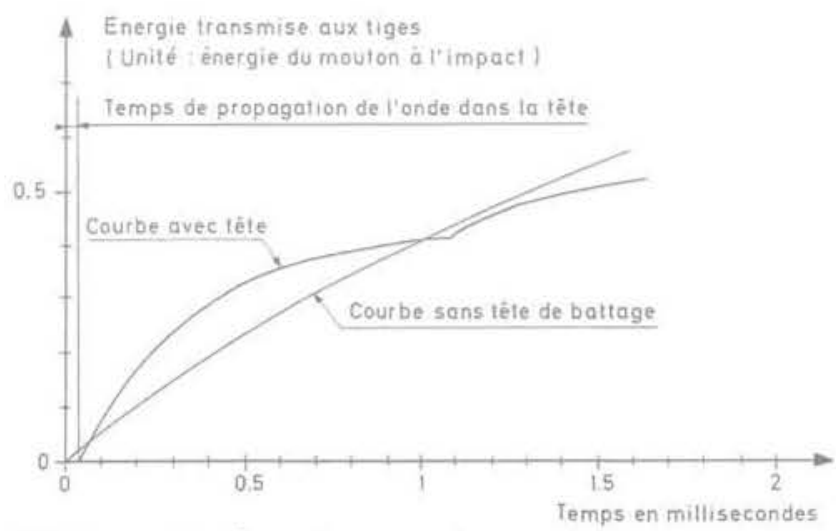

ศG. 10 Énergie transmise aux tiges. Energy induced into the drill string.
La réponse à la troisième question peut donc se formuler ainsi:

Une tête de battage modifie notablement l'intensité de l'onde de choc et peu la répartition dans le temps du transfert d'énergie; la part d'énergie perdue pour la pénétration est en principe augmentée.

Nous arrivons au terme de la deuxième partie; on peut constater sur un exemple que nous n'avons pas raisonné dans le vide. Sur les figures $11 \mathrm{a}$ et $11 \mathrm{~b}$, on peut voir:

- l'enregistrement de deux courbes de forces transmises lors du battage d'un pieu métallique; l'influence du casque est tout à fait visible et rappelle singulièrement notre exemple théorique sur l'étude de l'influence d'une tête de battage;

- les courbes de battages réelles, et celles qui ont été calculées avec des paramètres $\mathrm{K}$ et $\mathrm{q}$, uniques et ajustés pour le sol, la courbe d'intensité réelle de l'onde étant remplacée par une courbe théorique indiquée sur la figure 11a; la concordance est trop parfaite pour qu'il s'agisse d'une simple coincidence!

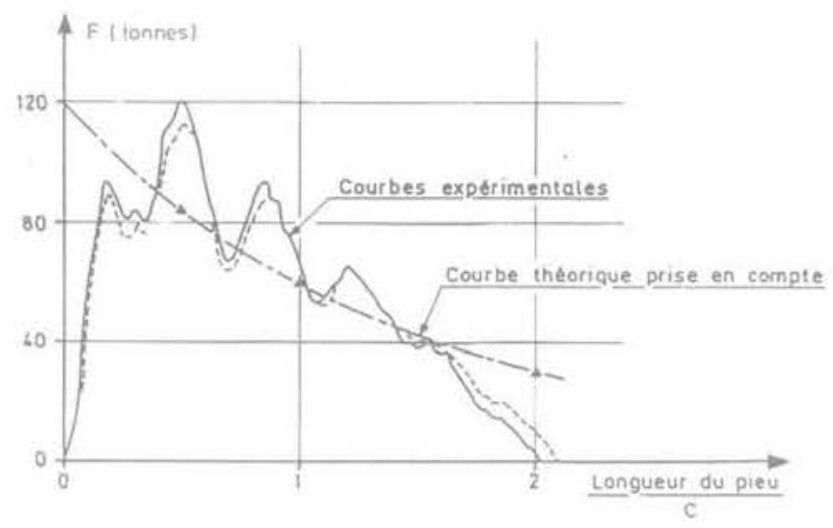

FiG. 11. Ondes de contrainte en tête de pieux. Stress wave on the pile head.

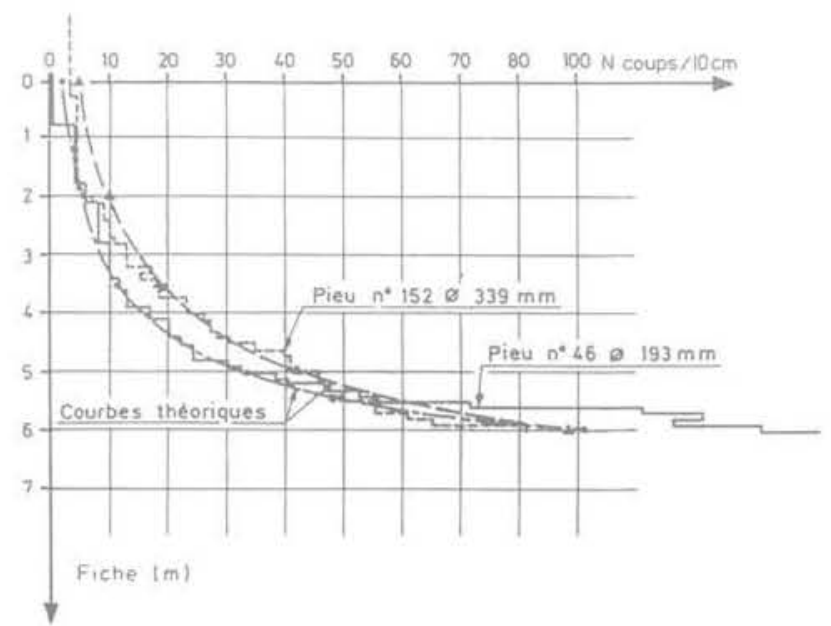

FIG. 116 Courbes de battage mesurées et calculées. Measured and calculated curves of driving advance. 


\section{Exemple de modélisation}

Nous sommes maintenant en mesure de bâtir notre modèle de pénétromètre dynamique et de le tester sur une coupe de terrain. Pour se ramener au cas d'une masse frappante sur une tige, sans tête de battage ni pointe élargie, on se propose de caractériser l'onde de choc par ses deux paramètres principaux seulement: la contrainte de pic ou intensité maximale, et l'énergie totale; cela revient à supposer que le transfert d'énergie en fonction du temps n'est guère modifié par la présence de la tête, ce que nous avons vérifié pour le cas qui nous occupe.

A la pointe, nous admettrons que la réaction du sol est proportionnelle à la surface de poinçonnement, mais que l'intensité de l'onde est modifiée suivant les règles établies pour un changement de section, ce qui est conforme à ce que nous avons vu plus avant.

Dans ces conditions, en prenant le pénétromètre dynamique type A de la norme AFNOR NF P 94114, nous pouvons prendre en compte les caractéristiques suivantes:

- masse frappante: masse $\mathrm{M}=64 \mathrm{~kg}$ : section $\mathrm{A}=0,0285 \mathrm{~m}^{2}$, hauteur $\mathrm{h}=0,2879 \mathrm{~m}$

- enclume: masse $\mathrm{m}=15 \mathrm{~kg}$ : section $\mathrm{a}_{\mathrm{T}}=$ $\mathrm{A} / 4=0,007125 \mathrm{~m}^{2}$, hauteur $=0,27 \mathrm{~m}$

- tige : masse au ml : 4,63 kg: section acier : $a_{1}=a_{1} / 12$, périmètre: $0,0425 \times 3,14=0,13345 \mathrm{~m}$, diamètre extérieur : $0,0425 \mathrm{~m}$

- pointe : section: $\mathrm{a}_{\mathrm{p}}=5 \mathrm{a}_{\mathrm{t}}=0,003 \mathrm{~m}^{2}$

En prenant une hauteur de chute $\mathrm{H}=0,75 \mathrm{~m}$, nous aurons:

$$
\begin{gathered}
V_{M}=\sqrt{2 g H}=3,836 \mathrm{~m} / \mathrm{s} \\
\frac{E}{c} V_{M}=\frac{2,1 \times 10^{11} \times 3,836}{5,1 \times 10^{3}}=158 \mathrm{MPa}
\end{gathered}
$$

La contrainte de pic dans les tiges, $\sigma_{\text {ort }}$ sera donnée par:

$$
\frac{E}{C} V_{M} \times \frac{4}{5} \times \frac{2 \times 12}{1+12}=233,35 \mathrm{MPa}
$$

La contrainte de pic dans la pointe sera:

$$
\sigma_{o p}=233,35 \times \frac{0,2 \times 2}{1,2}=77,78 \mathrm{MPa}
$$

La masse frappante et la vitesse d'impact équivalentes, qui donneraient la même énergie et la même contrainte de pic dans les tiges, sont données par:

$$
\begin{gathered}
V_{M}=\frac{c}{E} \sigma_{c t}=\frac{5,1 \times 10^{3} \times 233,35 \times 10^{6}}{2,1 \times 10^{11}}=5,67 \mathrm{~m} / \mathrm{s} \\
0,5 \times M \times(5,67)^{2}=64 \times 9,81 \times 0,75 \\
M=29,3 \mathrm{~kg}
\end{gathered}
$$

Les paramètres nécessaires pour le calcul sont donc les suivants:

$\mathrm{M}=29,3 \mathrm{~kg}$

$\mathrm{V}_{\mathrm{M}}=5,67 \mathrm{~m} / \mathrm{s}(\mathrm{H}=1,64 \mathrm{~m})$

$\mathrm{L}=6,33 \mathrm{~m}$ (longueur de tige ayant une masse de $29,3 \mathrm{~kg})$

$\mathrm{p}=0,13345 \mathrm{~m}$ (périmètre frottant des tiges)

$\mathrm{a}=0,59375 \times 10^{-3} \mathrm{~m}^{2}$ (section d'acier des tiges)
Pour le calcul de l'enfoncement, tout se passera comme si la valeur de $\mathrm{q}_{\mathrm{d}}$ du sol était multipliée par 3 par rapport à une pointe de même section que la tige, ou, ce qui revient au même, comme si la contrainte incidente dans la tige était divisée par 3.

Le calcul se fait par tranches successives de profondeur (pas de $1 \mathrm{~m}$ ou $0,5 \mathrm{~m}$ ) :

- calcul de la contrainte amortie, en fonction de la profondeur de fiche et du paramètre $\mathrm{K}$ pour le sol;

- calcul de l'enfoncement pour la première onde incidente, en fonction du paramètre $\mathrm{q}_{\mathrm{d}}$ pour le sol.

A chaque changement de couche, on calcule deux valeurs de l'enfoncement, ou plutôt de la courbe de battage (nombre de coups pour $10 \mathrm{~cm}$ d'enfoncement).

Reste à fixer un profil de sol. Nous prendrons quatre couches:

- de 0 à $5 \mathrm{~m}$ de profondeur: sable moyennement dense: $\mathrm{q}_{\mathrm{d}}=12 \mathrm{MPa}, \mathrm{K}=2 \mathrm{kPa} / \mathrm{m} / \mathrm{s}$;

- de 5 à $10 \mathrm{~m}$ de profondeur: argile molle : $\mathrm{q}_{d}=0,4 \mathrm{MPa}$, $\mathrm{K}=8 \mathrm{kPa} / \mathrm{m} / \mathrm{s}$;

- de 10 à $14 \mathrm{~m}$ de profondeur: sable dense : $\mathrm{q}_{\mathrm{d}}=25 \mathrm{MPa}$, $\mathrm{K}=5 \mathrm{kPa} / \mathrm{m} / \mathrm{s}$;

- de 14 à $20 \mathrm{~m}$ de profondeur: argile raide: $\mathrm{q}_{\mathrm{d}}=2,5 \mathrm{MPa}, \mathrm{K}=40 \mathrm{kPa} / \mathrm{m} / \mathrm{s}$.

En ce qui concerne le sable, on a pris un coefficient d'amortissement faible, n'ayant pas de résultats expérimentaux pour nous étalonner; par contre, pour les argiles, nous avons utilisé une corrélation qui a toujours bien fonctionné : $\mathrm{K}=\mathrm{C} / 3 \mathrm{~m} \mathrm{x} \mathrm{s}^{-1}$; ainsi, pour la couche d'argile molle, $\mathrm{C}_{4}=24 \mathrm{kPa}$ est cohérent avec $\mathrm{q}_{\mathrm{d}}=0,4 \mathrm{MPa}$ et $\mathrm{K}=8 \mathrm{kPa} / \mathrm{m} / \mathrm{s}$ (dans cet exemple, nous avons admis que l'incidence sur l'amortissement de la pointe élargie par rapport à la tige était négligeable).

Les résultats sont présentés sur la tableau I et les figures 12 et 13. Sur la figure 13, on a rassemblé:

- la courbe de $\mathrm{q}_{\mathrm{f}}$ en fonction de la profondeur: on peut penser qu'elle est peu différente de la courbe de pénétration statique $\left(\mathrm{q}_{\mathrm{c}} \# \mathrm{q}_{\mathrm{d}}\right)$;

- la courbe de la contrainte pic de l'onde incidente $\sigma_{n}$; l'enfoncement n'est possible que si $\left.\frac{2 \sigma_{i}}{3}\right\rangle q_{u}$; on peut suivre, en fonction de la profondeur, l'effet de l'amortissement;

- enfin, la courbe de q calculée d'après la formule des Hollandais, en prenant les enfoncements calculés: autrement dit, la courbe de présentation de l'essai sujvant la norme du pénétromètre dynamique type A.

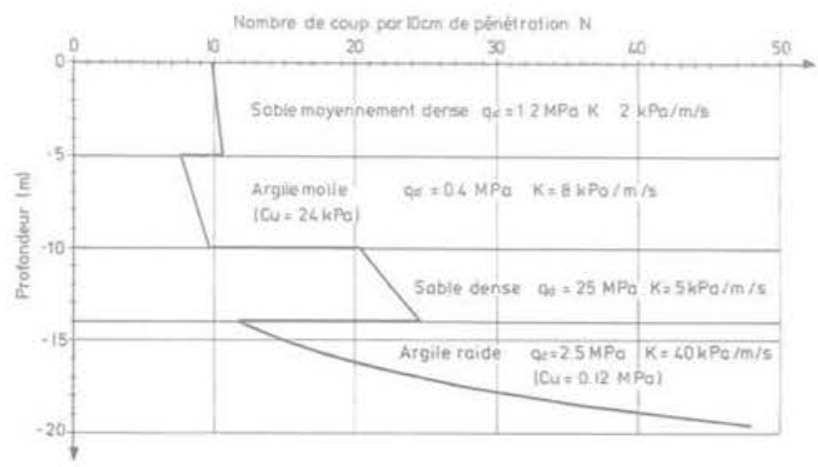

FG. 12 Courbe de battage calculée.

Calculated curves of driving advance. 


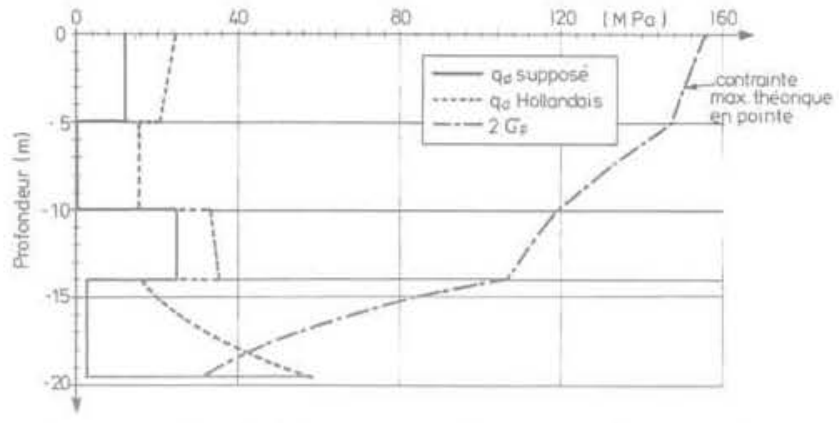

FIG. 13 Profils de $q_{d}$ et de la contrainte maximum en pointe.

$\mathrm{q}_{q}$ and maximum toe-stress versus depth.

On peut remarquer que l'incidence, dans la formule des Hollandais, de la prise en compte de la masse totale des tiges, injustifiable au point de vue théorique, est analogue à l'amortissement; mais cette incidence est soit exagérée, soit insuffisante. Par ailleurs, comme une part seulement de l'énergie est réellement utilisée pour la pénétration, la prise en compte de la formule des Hollandais conduit à majorer la résistance $q_{\alpha^{\prime}}$ du moins tant que l'influence de la masse des tiges ne devient pas prépondérante.
Remarquons encore que ce modèle peut facilement être adapté si plusieurs enfoncements successifs résultent des allers et retours d'une même onde.

En effet, la contrainte de pic de l'onde réfléchie est $q_{d}-\sigma_{j}$; si cette contrainte est négative et suffisamment grande en valeur absolue, il y aura un deuxième enfoncement au retour de l'onde. Pour un pas de calcul, il suffit donc de faire le test:

$$
\left.2\left(\sigma_{\mathrm{a}}-\mathrm{q}_{\mathrm{a}}\right)\left(\frac{\sigma_{\mathrm{a}}}{\sigma_{\mathrm{a}}}\right)^{2}\right) \mathrm{qd}
$$

Si cette inégalité est vérifiée, il y aura un deuxième enfoncement avec une contrainte $\sigma_{\mathrm{n}}=\left(\sigma_{\mathrm{a}}-\mathrm{q}_{\mathrm{p}}\right)\left(\sigma_{\mathrm{j}} / \sigma_{0}\right)^{2}$. et ainsi de suite, jusqu'à ce que l'inegalité ne soit plus vérifiée; on a donc une calcul itératif simple pour les faibles valeurs de $q_{j}$. Dans l'exemple présenté, si l'on corrige de cette façon la valeur initiale, le premier enfoncement est de $32,45 \mathrm{~mm}$ au lieu de 10,2 , et le nombre de coups $\mathrm{N}$ pour $10 \mathrm{~cm}$ d'enfoncement de 3 au lieu de 10; pour cette valeur initiale, il n'y a pas d'amortissement, et le calcul donne six allers et retours de l'onde provoquant des enfoncements successifs décroissants. Cette correction n'a pas été faite pour les résultats présentés ici.

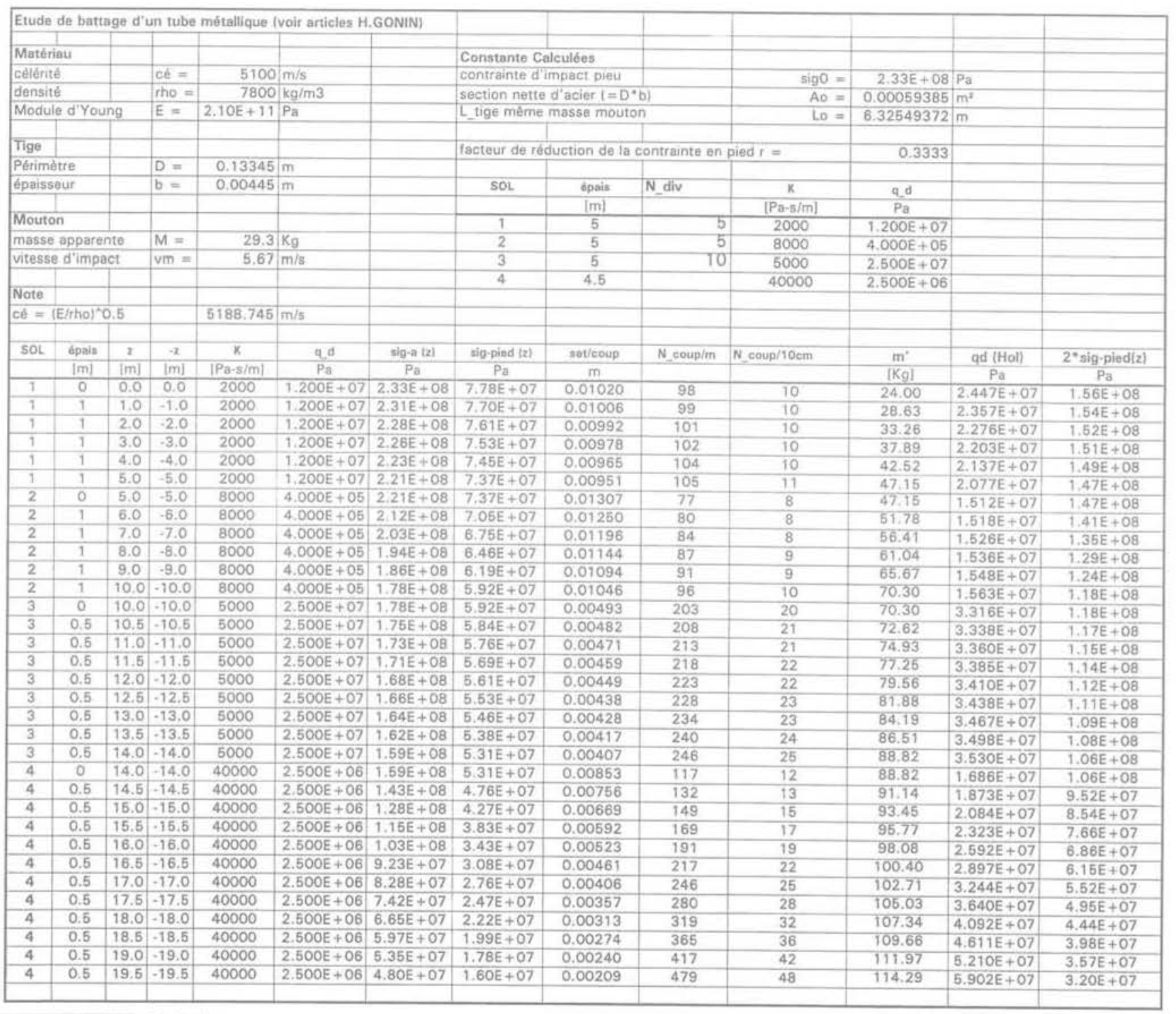

TABLEAU Calculs.

Calculations. 


\section{Le battage des pieux: encore trois questions !}

Le modèle simple proposé pour le pénétromètre dynamique ne permet pas de caractériser de manière univoque les sols testés, mais plutôt, en ramenant à l'essentiel, de tenir des raisonnements valides sur l'amortissement et la résistance à la pénétration dynamique dans les terrains traversés.

Il en est de même pour le battage des pieux, pour lequel il est indispensable de bien maîtriser l'énergie du choc et la manière dont elle est transmise au pieu. Sans parler des moyens de mesure et d'interprétation sophistiqués qui existent maintenant et qui sont surtout utilisés de manière courante en off-shore, nous abordons dans cette quatrième partie, en utilisant ce qui précède, trois nouvelles questions:

- quelle est la validité des formules de battage?

- comment définir simplement une «résistance au battage $), Q_{n}$ ?

- cette "résistance au battage " est-elle reliée de manière systématique à la résistance statique du pieu?

\section{1}

\section{Formules de battage}

Il est clair, et cela ne mérite pas la littérature qui y a été consacrée, que les formules de battage dérivées de la formule des Hollandais, quelles que soient les corrections apportées, n'ont aucun fondement physique, et qu'elles doivent donc être éliminées, y compris, malgré les habitudes, pour le pénétromètre dynamique !

Mais parmi les rares formules basées sur la théorie des ondes, nous analysons, ci-après, la formule de Kümmel (1984), proposée par Delmag et assez couramment utilisée.

Nous rappelons cette formule, avec les notations utilisées ici:

$$
\mathrm{s}=\Delta \mathrm{L} \frac{\left(\mathrm{Fmax}-\mathrm{Q}_{\mathrm{b}}\right)^{3}}{2 \mathrm{~F}_{\max }^{2} \cdot \mathrm{Q}_{\mathrm{b}}} \quad \Delta \mathrm{L}=\mathrm{MV}_{\mathrm{M}} / \mathrm{Z}=\mathrm{V}_{\mathrm{M}}(\mathrm{L} / \mathrm{c})
$$

$\mathrm{s}=$ refus mesuré; pour un mouton de référence, d'après ce qui précède, on a $F_{\max }=(E / c) V_{M}=p c V_{M}$ Kümmel suppose une forme triangulaire de londe de choc (voir Fig. 14) :

L'énergie d'une onde triangulaire de force maximale $F_{\max }$ est: $\xi=\frac{F_{\max }^{2} \mathrm{~L}}{3 \mathrm{EA}}$, à comparer à celle de l'onde de référence: $\frac{F_{\max }^{2} L}{2 E A}(A=$ section du pieu $)$.

La compression de cette onde triangulaire (reliée, comme nous l'avons vu, à la quantité de mouvement) est: $\frac{F_{\max }}{2 E A} \cdot L$, soit $\frac{\Delta \mathrm{L}}{2}(\Delta \mathrm{L}$ pour l'onde de référence).

Il semble que la formule utilisée pour la pénétration corresponde à l'énergie de l'onde hachurée sur la figure 14:

$$
\mathrm{s} \cdot \mathrm{Q}_{\mathrm{b}}=\frac{\left(\mathrm{F}_{\max }-\mathrm{Q}_{\mathrm{b}}\right)^{2}}{3 \mathrm{EA}} \cdot \mathbf{I} \quad \mathbf{I}=\left(\frac{\mathrm{F}_{\max }-\mathrm{Q}_{\mathrm{b}}}{\mathrm{F}_{\max }}\right) \cdot \mathrm{L} .
$$

d'où:

$$
\mathrm{S}=\frac{\left(\mathrm{F}_{\max }-\mathrm{Q}_{\mathrm{b}}\right)^{3}}{3 \mathrm{EAF} \mathrm{F}_{\max , Q_{\mathrm{b}}}} \cdot \mathrm{L}=\Delta \mathrm{L} \frac{\left(\mathrm{F}_{\max }-\mathrm{Q}_{\mathrm{b}}\right)^{3}}{3 \mathrm{~F}_{\max }^{2} \mathrm{Q}_{\mathrm{b}}}
$$

C'est la formule de Kümmel, à un facteur près ( 3 au lieu de 2 au dénominateur, sans explication rationnelle).

Mais le raisonnement n'est pas juste: la différence entre l'énergie de l'onde triangulaire et l'onde tronquée à $Q_{a}$ n'est pas égale à celle de l'onde hachurée; l'expression en est:

$$
\frac{F_{\max }^{2} L}{3 E A}-\frac{Q_{b}^{2}}{E A} \cdot \frac{\left(F_{\max }-Q_{b}\right)}{F_{\max }} \cdot L-\frac{Q_{b}^{2}}{3 E A} \cdot \frac{Q_{b}}{F_{\max }} \cdot L
$$

qui n'est pas égal à $\frac{\left(\mathrm{F}_{\max }-\mathrm{Q}_{\mathrm{b}}\right)^{3} \mathrm{~L}}{3 \mathrm{EA} \cdot \mathrm{F}_{\max }}$.

Par ailleurs, pour tenir compte de la présence d'un casque et de martyrs, Kümmel suppose que l'on applique à $F_{\max }$ un facteur de réduction, mais en gardant le produit $F_{\text {max }}$. L constant; or, pour que l'énergie soit conservée, c'est le facteur $F^{2}$ max. L qui doit être constant. C'est peut-être une maniére de tenir compte d'une perte d'énergie du fait de la présence du casque et des garnitures, mais il faut alors en souligner l'arbitraire!

Pour critiquable qu'elle soit, cette formule a cependant le mérite de donner une valeur plafonnée pour la résistance dynamique à la pénétration, assimilée à la force portante; ce n'est pas toujours le cas des autres formules !

Aucune formule de battage ne trouvant grâce à nos yeux, il nous reste donc à faire une proposition pour les remplacer!

\section{2}

\section{Définition d'une "résistance au battage " $Q_{b}$}

Supposant les paramètres de l'onde de référence, $\mathrm{F}_{\max }$ et $\mathrm{L}$, connus. A l'extrémité basse, l'onde amortie a pour paramètre $\mathrm{F}_{\mathrm{a}}=\lambda \mathrm{F}_{\text {thax }}, \lambda$ étant l'amortissement global à la profondeur considérée. La résistance à la pénétration dynamique est $\mathrm{Q}_{\mathrm{d}}=\mathrm{aq}_{\mathrm{d}}$. Nous proposons de définir simplement la résistance au battage $Q_{\mathrm{b}}$ :

$$
\mathrm{Q}_{\mathrm{b}}=\mathrm{F}_{\max }(1-\lambda)+\mathrm{Q}_{\mathrm{d}} \text { avec } \mathrm{Q}_{\mathrm{d}}<2 \lambda \mathrm{F}_{\max }
$$

Dans ces conditions, une mesure du refus élastique et du refus en fin de battage permettent de déterminer facilement $\lambda$ et $Q_{\mathrm{d}}$. En effet, comme nous l'avons remarqué plus haut, on $\mathrm{a}$, pour l'onde de référence, les deux formules:

$$
\begin{gathered}
\mathrm{S}=2 \lambda \frac{\mathrm{F}_{\max } \cdot L}{\mathrm{EA}}\left[1+\frac{\mathrm{Q}_{\mathrm{d}}}{2 \lambda \mathrm{F}_{\max }}\left(\ln \frac{\mathrm{Q}_{\mathrm{d}}}{2 \lambda \mathrm{F}_{\max }}-1\right)\right] \\
\mathrm{S}_{\mathrm{e}}=\frac{\mathrm{Q}_{\mathrm{d}} \cdot \mathrm{L}}{\mathrm{EA}}\left(1-\ln \frac{\mathrm{Q}_{\mathrm{d}}}{2 \lambda \mathrm{F}_{\max }}\right)
\end{gathered}
$$

$\lambda$ est donné par: $\mathrm{s}+\mathrm{s}_{\mathrm{e}}=2 \lambda \frac{\mathrm{F}_{\max } \cdot \mathrm{L}}{\mathrm{EA}}$

$Q_{d}$ est déterminé par la fonction $\mathrm{s}_{e}$ de $Q_{d}$.

Pour un battage d'essai, ces mesures peuvent être faites avec différentes fiches dans la couche portante et 


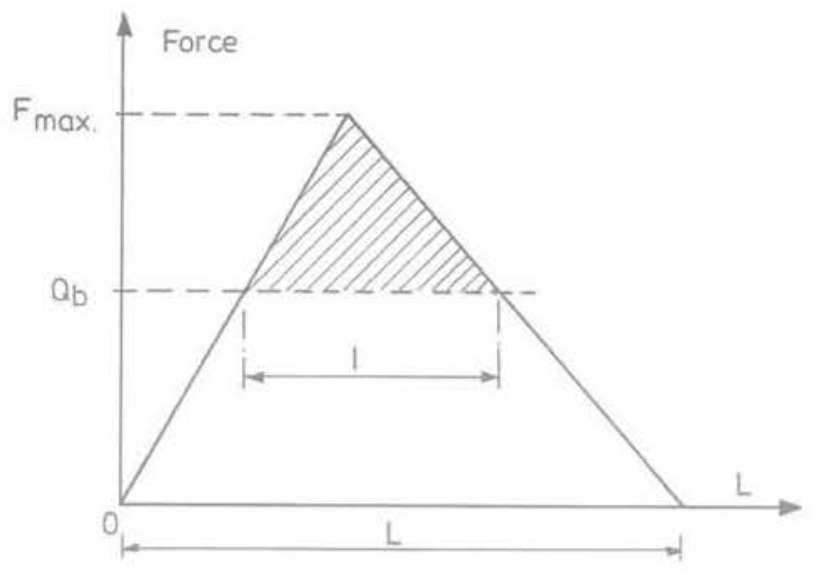

FIG 14 Onde triangulaire. Triangular stress-wave.

différentes énergies de battage ; à remarquer que le facteur $\lambda$ peut varier dans le temps pour une même fiche, par exemple entre la fin d'un battage et sa reprise le lendemain. Le suivi et l'interprétation des battages d'essai devraient, de cette façon, apporter d'utiles renseignements sur le comportement des pieux et des terrains au battage dans un site donné, et les critères de refus à respecter en fonction du site et de la portance demandée.

\section{3}

\section{Relation entre résistance au battage et résistance statique}

Il n'y a pas lieu d'être optimiste, en effet:

- l'amortissement de l'onde dépend largement du remaniement du sol autour du pieu, phénomène essentiellement transitoire et même aléatoire (liquéfaction partielle ou non, surpressions ou dépressions interstitielles gênérées dans les sols saturés);

- cet amortissement correspond à un phénomène physique complètement différent de la mobilisation d'une résistance au cisaillement avant rupture; il n'affecte pas non plus les mêmes surfaces: pour l'amortissement, la longueur d'onde intervient;

- en ce qui concerne la résistance dynamique en pointe, le phénomène est sans doute dépendant de la vitesse de pénétration; on ne peut même pas dire si, lorsque cette vitesse tend vers zéro, la résistance $Q_{\text {, }}$ tend vers $Q_{s}$ car vitesse et résistance à la pénétration sont reliées én dynamique.

Malgré ces remarques décourageantes, il ne faut certainement pas abandonner l'idée d'apprécier une "résistance au battage», au contraire! Mais tirons-en des conclusions sur la manière d'utiliser l'information ainsi recueillie; n'oublions pas, en particulier, qu'il ne s'agit pas de déterminer des caractéristiques intrinsèques des sols traversés mais des paramètres reliés à la sollicitation particulière due à l'enfoncement par battage. Encore faut-il, pour établir des corrélations valides, choisir de bons paramètres, simples, peu nombreux, et ayant une réelle signification physique.

\section{5}

\section{Conclusion}

Cette étude est un plaidoyer pour introduire à la fois rationalité et pragmatisme dans un domaine où il semble qu'on n'ait le choix, à l'heure actuelle, qu'entre les mesures et analyses sophistiquées et une aveugle confiance dans le savoir-faire sur le chantier. Pour commencer, une exploitation plus rationnelle des résultats des essais de pénétration dynamique ne pourrait qu'améliorer les choses; la mesure systématique du refus êlastique en cours de battage et celle de la vitesse à l'impact de la masse frappante au moment du choc constitueraient un progrès appréciable: on doit, par exemple, souligner la tentative pleine de succès du PANDA, dont l'étude rationnelle devrait, nous semblet-il, être approfondie. Pour le battage des pieux. l'enregistrement des paramètres essentiels (vitesse de la masse frappante à l'impact, refus et refus élastique par coup, cadence) devrait être la norme, Ce qui est proposé ici, c'est une méthode d'analyse simple et rapide, basée sur des mesures faciles à réaliser sans équipement sophistiqué, à la portée de tout technicien s'étant intéressé à la question.

\section{Bibliographie}

Bustamante M. et al. - Vérification expérimentale des formules de battage. Symposium International sur la pénétrabilité et le fonçage des pieux, San Francisco, aoùt 1985 , vol. 1, 1985.

Fischer H.C. - Stress wave theory for pile driving applications, 2nd international Corference on the application of stress wave theory on piles Stockholm. Suède, 1984.

Gonin H. - Etude théorique du battage des corps élastiques élancés, Annales ITBTP
- Série sols et fondations, $n^{\circ} 155,1978$.

Gonin H. - Réflexions sur le battage de pieux, Revue française de géotechnique, $n^{\circ}$ 9, 1979

Gonin H. - Rapport génẻral sur la pénétrabilité et le fonçage des pieux, comptes rendus du Symposium International sur la pénétrabilitè et le fonçage des pieux. San Francisco, août 1985, vol. 1, 1985.

Heritier B, Paquet J. - Détermination de la capacitê portante des pieux par les méthodes dynamiques, id. San Francisco, août 1985, vol. 1. 1985.

Kümmel F. - The Kümmel method for calculation of impact forces in piles, 2nd Int. Conf, on the application of stresswave theory on piles, Stockholm, Suede, 1984.

De Saint-Venant - Mémoire sur le choc longitudinal de deux barres élastiques, J. de mathématiques, 2 ser. XII, 1867 , p. 237-376. 